\title{
Unsteady Computations of a Jet in Crossflow with Ground Effect
}

\author{
Shishir Pandya ${ }^{*}$ \\ NASA Ames Research Center, Moffett Field, CA 94035 \\ Scott Murman ${ }^{\#}$ \\ ELORET, Moffett Field, CA 94035 \\ and \\ Sankaran Venkateswaran ${ }^{*}$ \\ University of Tennessee, Tullahoma, TN
}

\section{Abstract}

A numerical study of a jet in crossflow with ground effect is conducted using OVERFLOW with dual time-steppig and low Mach number preconditioning. The results of the numerical study are compared to an experiment to show that the numerical methods are capable of capturing the dominant features of the flow field as well as the unsteadiness associated with the ground vortex.

\section{Introduction}

The study of a jet impinging on a ground in crossflow has been the subject of both experimental ${ }^{[1]}$ and numerical ${ }^{[2,3]}$ studies in the past due to interest in V/STOL vehicle behavior in near-hover conditions. Of interest are issues such as the extent, location, strength, and frequency of the ground vortex, which are crucial for the safety of the ground crew and for assessing hot gas ingestion effects on aircraft engine operation.

The flowfield associated with the present test case has several important features. When the jet impinges on the ground, the air flows radially outward from the point of impact. A ground vortex is created in the shape of a horseshoe due to the presence of a crossflow. Numerical studies of a jet in crossflow ${ }^{[2]}$ and V/STOL aircraft ${ }^{[3,4,5]}$ have been successfully performed. In the numerical investigation of Ref. 2 , the flow did not exhibit unsteady behavior. In order to make it "go unsteady," the jet needed to be pulsed. In the studies of Refs. 3, 4, and 5, experimental verifications of the ground vortex predicitons were not available. In the current paper, the focus is on unsteady simulations of the jet in crossflow with ground effect for comparison with the experiments of Cimbala et. al. ${ }^{[1]}$

\footnotetext{
${ }^{¥}$ Research Scientist, Member AIAA

\# Senior Research Scientist

${ }^{*}$ Research Professor, Member AIAA
} 
The unsteady jet computations are obtained using the OVERFLOW code along with preconditioned dual-time scheme. ${ }^{[6][11]}$ Preliminary results shown in this abstract demonstrate that the gross features of the flowfield are well represented and the unsteady frequency associated with the ground vortex agrees well with the experiment. The final version of the paper with include grid refinement studies as well as detailed parametric studies varying the crossflow velocity, jet velocity, jet diameter, and jet height.

The following section describes the numerical method used for the simulation of the jet. Sec. 4describes the grid system and the problem configuration. In Sec.5, a numerical solution of the jet in Crossflow and ground effect is shown and the details of the solution are discussed.

\section{Technical Approach}

The OVERFLOW ${ }^{[6]}$ Navier-Stokes flow solver is used to perform the present computations. The diagonalized approximate factorization algorithm of Ref. 8 is used in conjuction with dual timestepping to get time-accurate results. A low Mach number preconditioner based on Refs. 9, and 10 is used to capture the Mach 0.013 crossflow accurately.

The implementation of a low Mach number preconditioner for unsteady flows in OVERFLOW is discussed in detail in Ref. 11 . For completeness, a summary of the formulation follows.

\subsection{Low Mach Number Preconditioning and Dual time-stepping}

The preconditioned dual-time stepping method in OVERFLOW is based on the dual time-stepping methods presented in references [7][10]. The Navier-Stokes equations can be written in conservative form as

$$
\Gamma_{p} \frac{\partial Q}{\partial \tau}+\frac{\partial Q}{\partial t}+\frac{\partial E}{\partial x}+\frac{\partial F}{\partial y}+\frac{\partial G}{\partial z}=L(Q)
$$

where $Q=[\rho \rho u \rho \rho w e]^{T}$ is the vector of unknowns, E, F, and $\mathrm{G}$ are the inviscid fluxes and $L(Q)$ represents the viscous terms. The term $\Gamma_{p}$ is the preconditioning matrix. The artificial time term $\frac{\partial Q}{\partial \tau}$ is introduced to the governing equations in order to provide a relaxation (sub-iteration) procedure between physical time steps.

Termed dual time-stepping, the subiteration process is important for ensuring efficient convergence of the time dependent solutions at each physical time step. In combination with preconditioning, it also allows the use of larger time steps to efficiently capture the low frequency "puffing" of the ground vortex. The preconditioning formulation is also important for insuring accuracy at the low Mach number conditions of the crossflow.

In generalized coordinates, the Navier-Stokes equations are discretized with first order accurate Euler implicit discretization for the artificial time term, second order backwards difference discretization for the physical time terms and central difference discretization for the spatial terms to obtain

$\left(I+\Delta \tau \Gamma_{e} S_{p}^{-1} A^{k} \delta_{\xi}+\Delta \tau \Gamma_{e} S_{p}^{-1} B^{k} \delta_{\eta}+\Delta \tau \Gamma_{e} S_{p}^{-1} C^{k} \delta_{\zeta}\right) \Delta \hat{Q}=\Gamma_{e} S_{p}^{-1} R^{k}$ 
where $\hat{Q}=J^{-1} Q, S_{p}=\Gamma_{p}+\frac{3 \Delta \tau}{2 \Delta t} \Gamma_{e}, \Gamma_{e}=\frac{\partial Q}{\partial Q_{p}}$ (a transformation matrix between conservative and primitive variables), $\mathrm{A}, \mathrm{B}$, and $\mathrm{C}$ are the flux Jacobians and

$$
R^{k}=-\Delta \tau\left(\frac{3 \hat{Q}^{k}-4 \hat{Q}^{n}+\hat{Q}^{n-1}}{2 \Delta t}+\delta_{\xi} \hat{E}^{k}+\delta_{\eta} \hat{F}^{k}+\delta_{\zeta} \hat{G}^{k}-L(Q)\right) \text {. }
$$

The variable $n$ is the time step counter while the variable $k$ is the sub-iteration counter. Finally, $\Delta Q=Q^{K+1}-Q^{k}$. A diagonalized approximate factorization algorithm[8] is used for the solution of this equation. When converged in artificial time, this method is formally second order accurate.

The spalart-Allmaras turbulence model is used for the present computations.

\section{Problem Configuration}

The configuration is designed to match the conditions in the experiment of Ref. 1 . The experiment was conducted in a test section where the top and a bottom walls are 21 inches apart. A jet tube is inserted through the top wall and is 12 inches in length leaving a 9 inch gap before the jet hits the ground. The jet exits a 3 inch diameter tube at a speed of $150 \mathrm{ft} / \mathrm{s}$ or Mach 0.13 . The crossflow speed is $15 \mathrm{ft} / \mathrm{s}$ which is equivalent to a Mach number of 0.013 . The Reynolds number based on the crossflow speed is 80000 .

The OVERFLOW code solves the Reynolds averaged Navier-Stokes equations on structured grids. For the purpose of the numerical simulation, the jet profile was set to the idealized slug flow at the top of the grid. A boundary layer was then allowed to develop in the jet tube and this was considered adequate for the purpose of obtaining a valid velocity profile at the exit of the jet tube.

\subsection{Grid system}

Unlike a full aircraft simulation, the single jet has the advantage that there is no complex geometry involved. Thus, a structured grid in a single cylindrical block, shown in Fig. 1 can be used for the present numerical simulation. The structured grid has the dimensions of 99(radial)x51(vertcal) $\times 51$ (circumferential) for a total of approximately 250,000 grid points. The jet is placed at the center of the mesh. This same jet and the wall of the jet tube are depicted in a cross section of the grid( $99 \times 51)$ shown in Fig. 2 to illustrate the problem and the boundaries. This cross section shows half of an XY cut through the grid such that the center of the cylindrical mesh corresponds to the right hand side of the figure. The incoming flow enters through the left boundary in Fig. 2 and the jet tube and the jet are on the right.

\section{Results}

The typical flow field corresponding to a jet impinging on a ground is expected to consist of the jet hitting the ground and splaying in all directions. However, due to the crossflow pushing the forward moving part of the splayed jet backwards, a ground vortex develops in front of the jet as shown in Fig. 3 . This ground vortex is blown backwards by the crossflow thus wrapping itself around the jet in a horseshoe shape when viewed from above. A particle trace of the jet and the 
crossflow is used to show this horseshoe shape in Fig. 4 . As indicated in Ref. 1, the "puffing" of this ground vortex becomes the primary source of the unsteadiness.

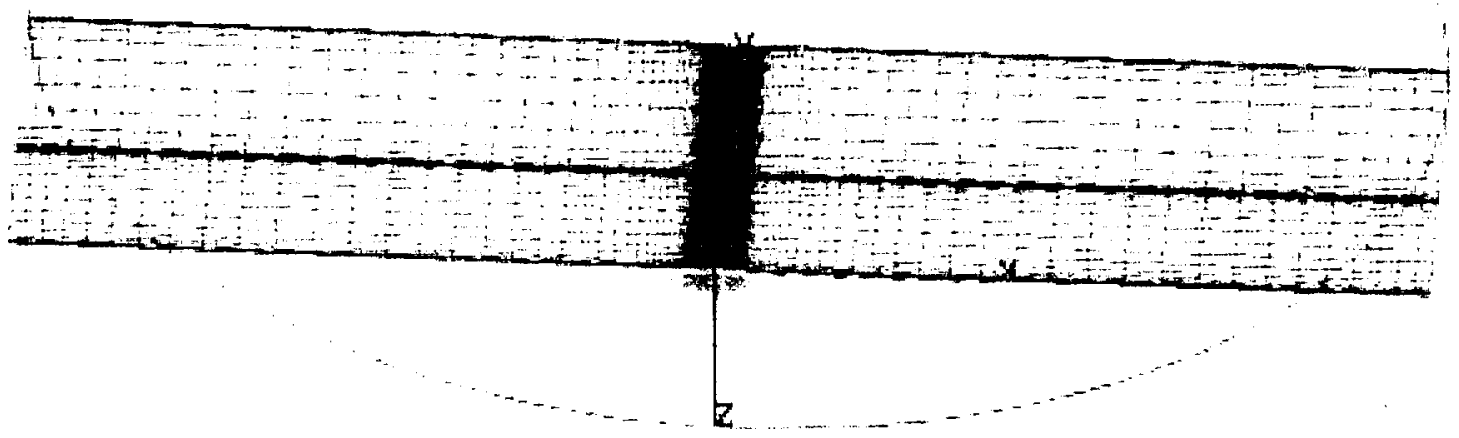

Figure 1: Oblique view of the grid

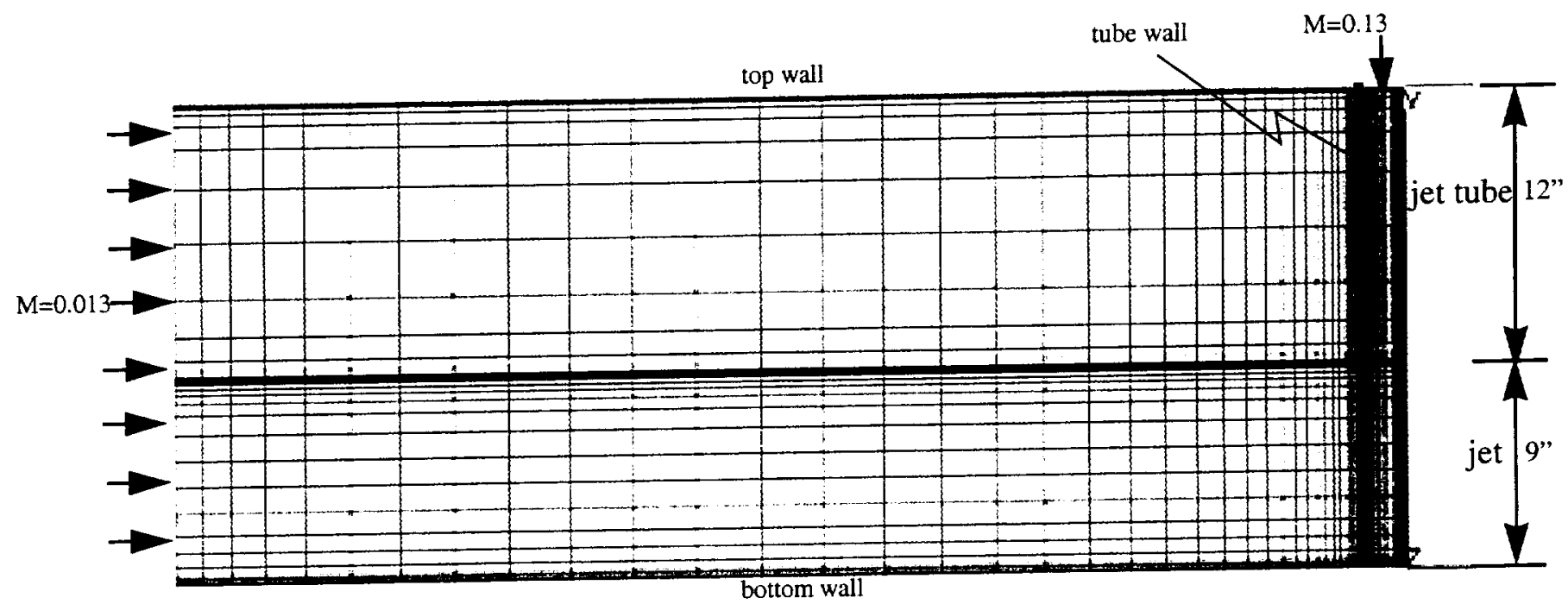

Figure 2: A cross-section of the grid 
A common method to verify if a numerical solution has developed unsteadiness is to plot the residual of the discretized equations as a function of the time step. For steady flows, the residual tends to drop several orders of magnitude and then settle to a single value. In ideal cases, the residual is expected to go to machine zero. Unsteadiness in the flow field is clearly indicated by a residual that instead of settling to a single value, continues to increase and decrease. If the residual pattern has a specific frequency, it is most likely associated with some unsteady feature of the fulfilled. The residual history for the present test case is shown in Fig. 5 . The repeating pattern is seen in this history and corresponds to a frequency of approximately $1.4 \mathrm{~Hz}$. The observed frequency in the experiments of Cimbala et. al. corresponded to between 1 and $8 \mathrm{~Hz}$ with the frequency for the case presented here being approximately $3.5 \mathrm{~Hz}$.

\section{Summary}

The purpose of this paper is to numerically simulate this three dimensional unsteady flow in order to verify that the features in the flow field can be obtained numerically. Furthermore, computations will be performed for several conditions in order to compare the unsteady frequency in the numerical solution to the frequency in the experiment of Cimbala et. al.

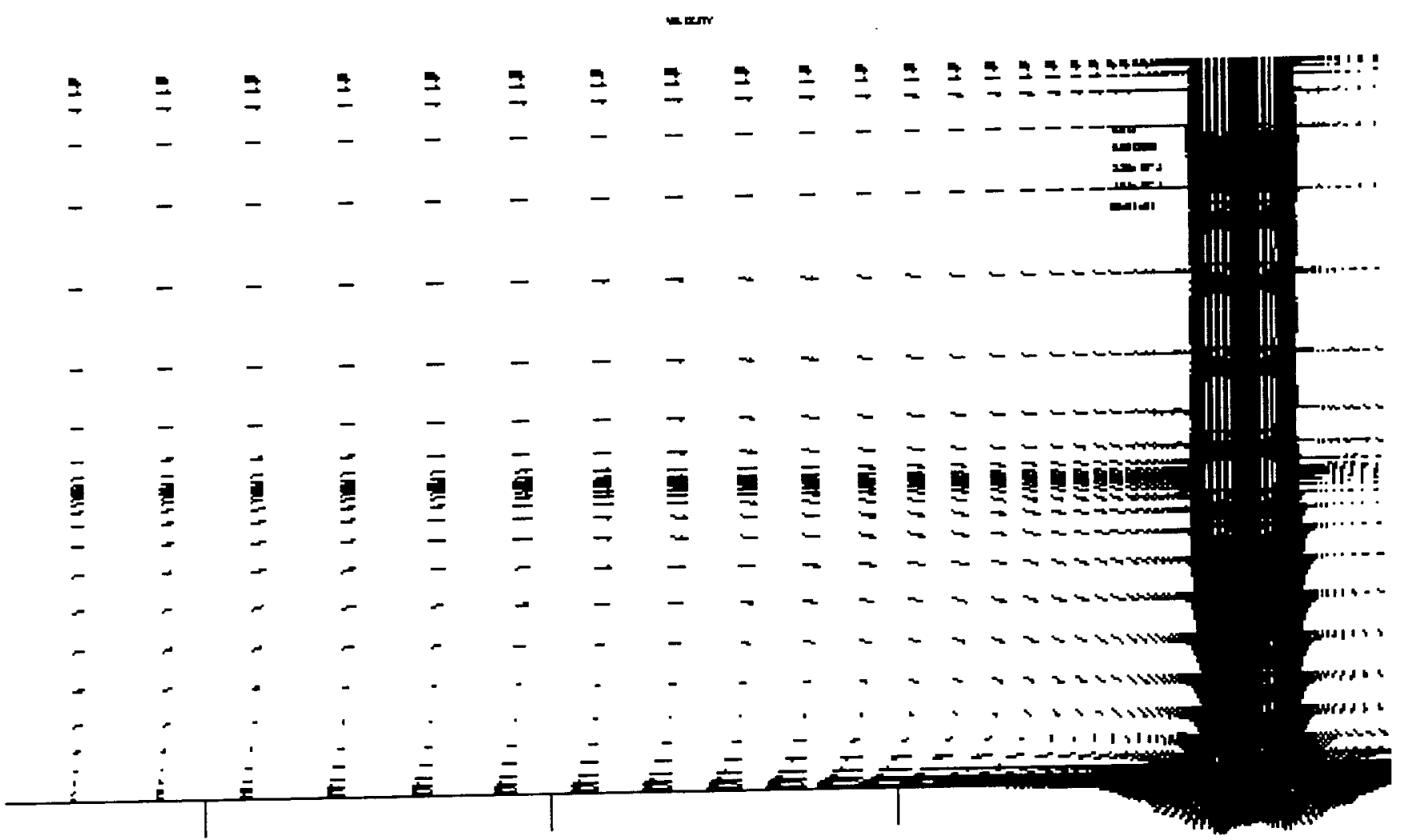

Figure 3: A vector plot of the jet and the ground vortex 


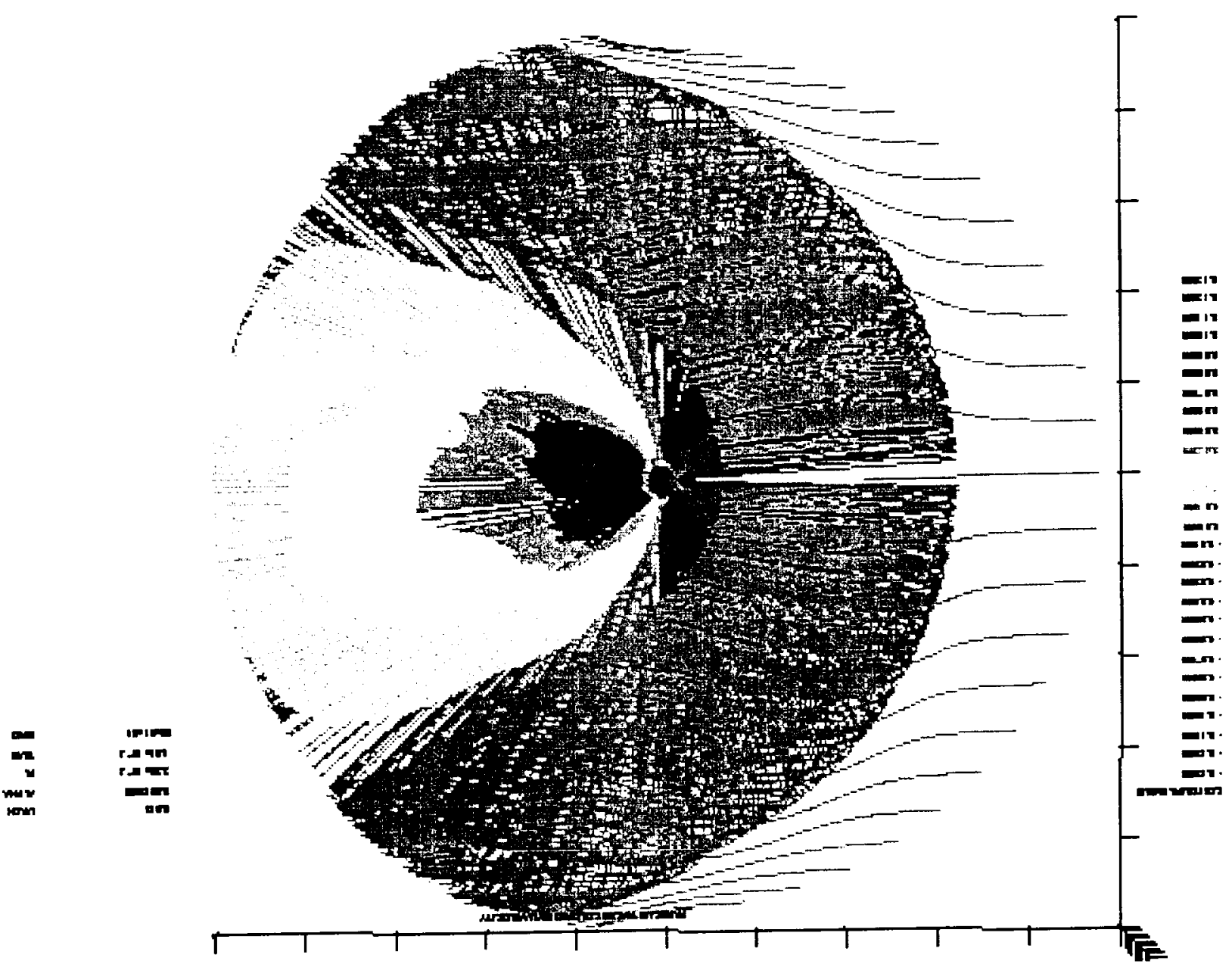

$6 L Z Z-\angle 8 \cdot$ oN $12 \mathrm{de} \mathrm{d}$ VVTV

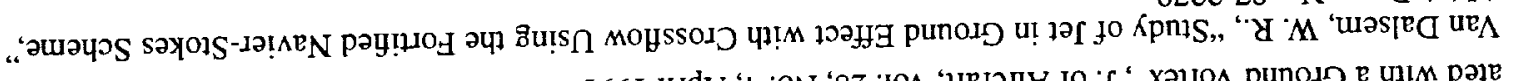

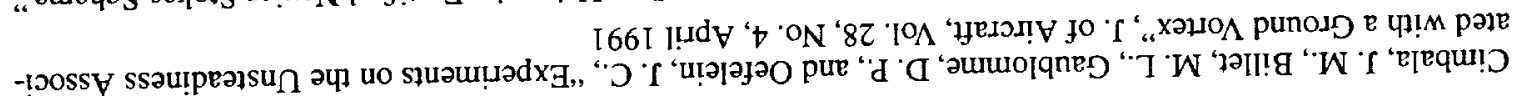
[z]

Sววนวдадау $L$

- SMOH Jo sSelo

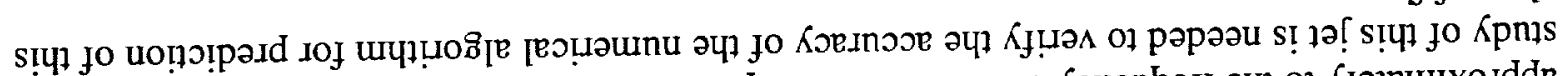

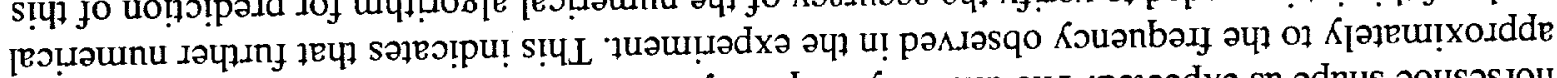

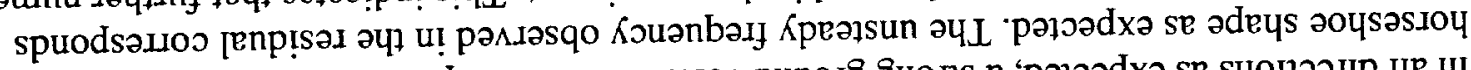

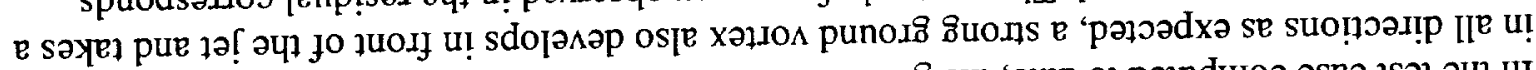

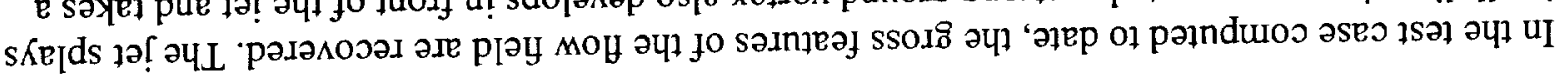




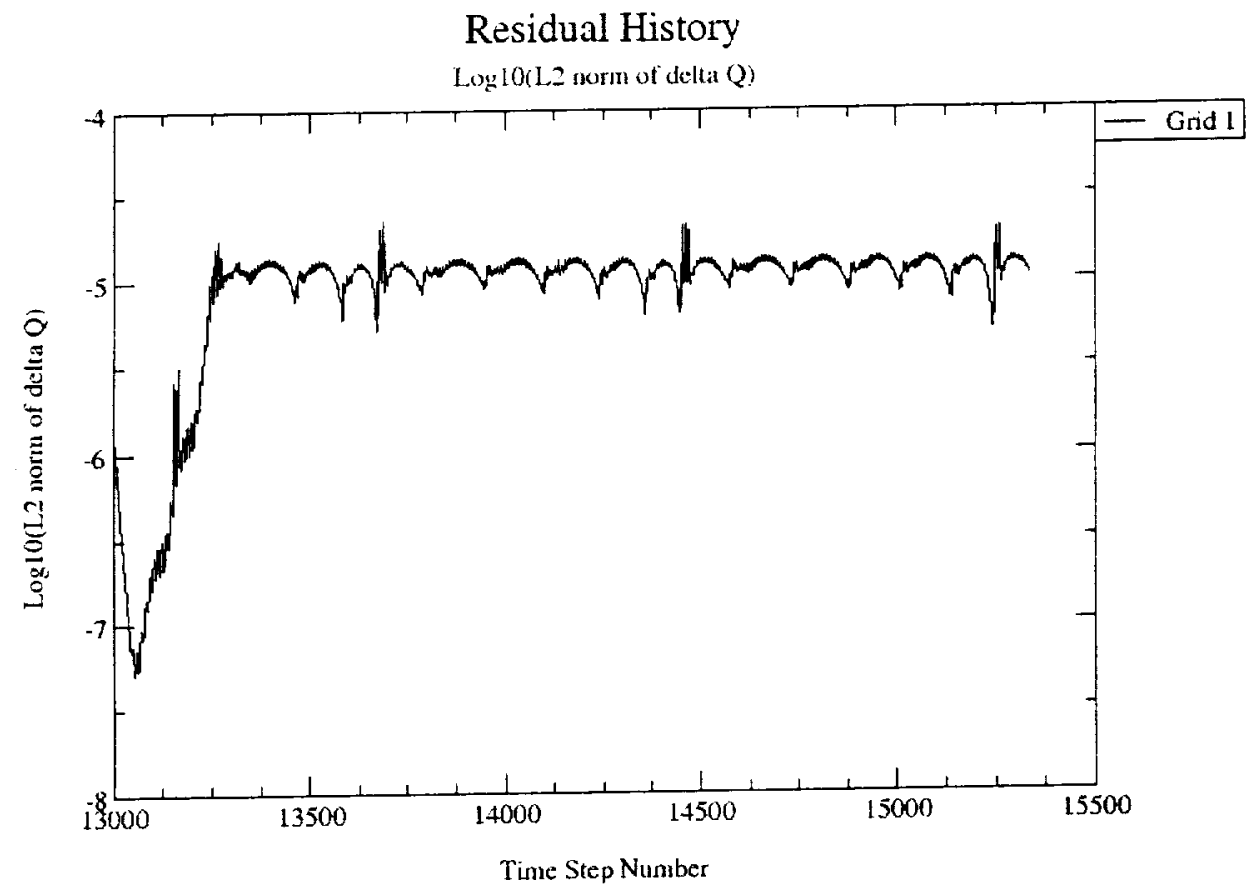

Figure 5: Residual history of the unsteady solution

[3] Smith, M. H., Chawla, K., and Van Dalsem, W. R., "Numerical Simulation of a Complete STOVL Aircraft in Ground Effect," AIAA Paper No. 91-3293

[4] Chaderjian, N. M., Pandya, S., Ahmad, J., and Murman, S. M., "Parametric Time-Dependent Navier-Stokes Computations for a YAV-8B Harrier in Ground Effect," AIAA Paper No. 2002-0950

[5] Pandya, S. A., Chaderjian, N., and Ahmad, J., "Parametric Study of a YAV-8B Harrier in Ground Effect Using Time-Dependent Navier-Stokes Computations," AIAA Paper No. 2002-3056

[6] Buning, P. G., Jespersen, D. C., Pulliam, T. H., Chan, W. M., Slotnick, J. P., Krist, S. E., and Renze, K. J. "OVEFLOW User's manual," NASA.

[7] Pulliam, Thomas H., "Time accuracy and the use of implicit methods," AIAA Paper No. 93-3360

[8] Pulliam, T. H., and Chausee, D. S., "A diagonal form of an implicit approximate-factorization algorithm," Journal of Computational Physics, Vol. 39, No. 2, 1981, pp. 347-363

[9] Buelow, P. E. O., Schwer, D. A., Feng, J., and Merkel, C. L., "A preconditioned Dual-Time, Diagonalized ADI Scheme for Unsteady Computations," AIAA Paper No. 97-2101

[10] S. Venkateswaran and Merkle, C. L., "Dual time" stepping and preconditioning for unsteady computations," AIAA Paper No. 95-0078

[11] Pandya, S. A., Venkateswaran, S., and Pulliam, T., "Implementation of Preconditioned Dual-Time Procedures in OVERFLOW," AIAA Paper No. 2003-0072, To be presented at 41st AIAA Aerospace Sciences Meeting and Exhibit, Reno, NV, 6-9 Jan 2003 